\title{
Branched Chain Amino Acids Are Associated with Insulin Resistance Independent of Leptin and Adiponectin in Subjects with Varying Degrees of Glucose Tolerance
}

\author{
Margery A. Connelly, PhD, MBA, Justyna Wolak-Dinsmore, PhD, and Robin P.F. Dullaart, MD, PhD ${ }^{2}$
}

\begin{abstract}
Background: Branched chain amino acids (BCAA) may be involved in the pathogenesis of insulin resistance and are associated with type 2 diabetes mellitus (T2DM) development. Adipokines such as leptin and adiponectin influence insulin resistance and reflect adipocyte dysfunction. We examined the extent to which the association of BCAA with insulin resistance is attributable to altered leptin and adiponectin levels in individuals with varying degrees of glucose tolerance.

Methods: BCAA were measured by nuclear magnetic resonance, whereas leptin and adiponectin were measured by immunoassay, in subjects with normal fasting glucose $(n=30)$, impaired fasting glucose $(n=25)$, and T2DM $(n=15)$. Insulin resistance was estimated by homeostasis model assessment (HOMAir).

Results: BCAA were higher in men than in women $(P<0.001)$ and tended to be higher in T2DM subjects $(P=0.10)$ compared to subjects with normal or impaired fasting glucose. In univariate regression analysis, BCAA were correlated with HOMAir $(r=0.46 ; P<0.001)$ and inversely with adiponectin $(r=-0.53 ; P<0.001)$ but not with leptin $(r=-0.08 ; P>0.05)$. Multivariable linear regression analysis, adjusting for age, sex, T2DM, and body mass index (BMI), demonstrated that BCAA were positively associated with HOMAir $(\beta=0.242$, $P=0.023$ ). When BCAA, leptin, and adiponectin were included together, the positive relationship of HOMAir with BCAA $(\beta=0.275, P=0.012)$ remained significant.

Conclusions: Insulin resistance was associated with BCAA. This association remained after adjusting for age, sex, T2DM, BMI, as well as leptin and adiponectin. It is unlikely that the relationship of insulin resistance with BCAA is to a major extent attributable to effects of leptin and adiponectin.
\end{abstract}

Keywords: branched chain amino acids, nuclear magnetic resonance spectroscopy, insulin resistance, adipose tissue dysfunction, type 2 diabetes mellitus

\section{Introduction}

B RANCHED CHAIN AMINO ACIDS (BCAA) have been shown to be elevated in metabolic disorders associated with insulin resistance, such as obesity, metabolic syndrome, and type 2 diabetes mellitus (T2DM). ${ }^{1-4}$ Potential mechanisms for enhanced circulating BCAA concentrations include excess dietary consumption, elevated production by gut microbes, and decreased BCAA catabolism in muscle and adipose tissue. ${ }^{1,3-5}$ It has been hypothesized that BCAA may in fact be a causal factor in the development of insulin resistance and T2DM by contributing to the overload of mitochondria with lipid substrates, which subsequently leads to mitochondrial stress and impaired insulin action. ${ }^{1,3}$ Consistent with these observations, BCAA may be predictive of diabetes development. ${ }^{6-8}$ BCAA levels are, moreover, responsive to therapeutic interventions that enhance insulin sensitivity. ${ }^{9}$ Therefore, measurement of circulating BCAA may be useful for determining metabolic and adipose tissue dysfunction in advance of chronic disease development.

It is well known that the adipokines, leptin and adiponectin, are altered in obesity, metabolic syndrome, and T2DM. ${ }^{10,11}$ While both adipokines are secreted by adipose tissue, leptin levels are higher and adiponectin levels are

\footnotetext{
${ }^{1}$ LipoScience, Laboratory Corporation of America ${ }^{\circledR}$ Holdings, Raleigh, North Carolina.

${ }^{2}$ Department of Endocrinology, University Medical Center Groningen, University of Groningen, Groningen, the Netherlands.

(C) Margery A. Connelly, et al., 2017; Published by Mary Ann Liebert, Inc. This Open Access article is distributed under the terms of the Creative Commons Attribution Noncommercial License (http://creativecommons.org/licenses/by-nc/4.0/) which permits any noncommercial use, distribution, and reproduction in any medium, provided the original author(s) and the source are credited.
} 
lower in obese subjects. ${ }^{12,13}$ Leptin and adiponectin play key roles in adipocyte differentiation. ${ }^{14-16}$ Moreover, the leptin/ adiponectin ratio is related to systemic inflammation and is a determinant of insulin resistance in nondiabetic individuals, highlighting the contribution of adipose tissue dysfunction to the pathogenesis of diminished insulin action. ${ }^{17,18}$

The aim of this study was to interrogate the extent to which the potential association of insulin resistance with circulating BCAA is attributable to altered leptin and adiponectin levels in individuals with varying degrees of glucose tolerance.

\section{Materials and Methods}

\section{Patients}

The Medical Ethics Committee of the University Medical Center Groningen approved the study protocol. Participants, aged $>18$ years, were recruited by newspaper advertisement. Written informed consent was obtained. Glucose tolerance status was classified as normal fasting plasma glucose (FPG $<5.6 \mathrm{mM}$ ), impaired fasting glucose (FPG $\geq 5.6$ and $\leq 6.9 \mathrm{mM}$ ), and T2DM (FPG $\geq 7.0 \mathrm{mM}$; previously determined non-FPG $\geq 11.1 \mathrm{mM}$ ), according to NCEP-ATPIII criteria and guidelines from the Dutch College of General Practitioners. T2DM had been previously diagnosed by general practitioners. Subjects who used insulin, oral glucose-lowering drugs, lipid-lowering drugs, or antihypertensive medication were not allowed to participate in the present study. This was done to avoid confounding of medication use on insulin resistance and other relevant variables. From an initially selected group of 103 subjects, 33 were excluded because they used glucose-lowering drugs or antihypertensives. Subjects had been given dietary advice as part of their diabetic treatment; their nutrient intake was not standardized before entry in the study. Current smokers were also excluded, as were subjects with history of cardiovascular or chronic kidney disease, liver or thyroid dysfunction.

All participants were evaluated after an overnight fast. Blood pressure was measured as described. ${ }^{18}$ Body mass index (BMI; $\mathrm{kg} / \mathrm{m}^{2}$ ) was calculated as weight divided by height squared. Homeostasis model assessment of insulin resistance $\left(\mathrm{HOMA}_{\mathrm{ir}}\right)$ was used to estimate insulin resistance (fasting insulin $(\mathrm{mU} / \mathrm{L}) \times$ fasting glucose $(\mathrm{mM}) / 22.5)$.

\section{Laboratory analysis}

EDTA plasma was collected and stored at $-80^{\circ} \mathrm{C}$ until analysis. Plasma glucose, total cholesterol, HDL-cholesterol, triglycerides, insulin, leptin, and adiponectin were assayed as described. ${ }^{19}$ Intra-assay and interassay coefficient of variations (CVs) were $<6 \%$ and $<8 \%$, respectively.

\section{Nuclear magnetic resonance data acquisition and BCAA signal deconvolution analysis}

Nuclear magnetic resonance spectra were acquired using EDTA plasma on a Vantera ${ }^{\circledR}$ Clinical Analyzer as previously described. $^{20,21}$ For BCAA analysis, an optimized deconvolution algorithm was implemented, which simultaneously models the methyl signals for the lipoproteins, proteins, and BCAA between 0.718 and $1.02 \mathrm{ppm}$ in the serum spectrum. Concentrations were determined using non-negative linear least squares by determining the signal area and multiplying by particle conversion factors. Intra-assay and interassay CVs for BCAA were $3.2 \%$ and $3.3 \%$, respectively.

\section{Statistical analysis}

SPSS (version 23.0; SPSS, Inc., Chicago, IL) was used for data analysis. Results are expressed as mean \pm SD or as median (interquartile range). Variables between the three groups were compared by ANOVA with the Bonferroni method to correct for multiple comparisons or by the chisquare test. Univariate relationships were assessed with Pearson's correlation coefficients. Because of skewed distributions, logarithmically transformed values of $\mathrm{HOMA}_{\mathrm{ir}}$, leptin, and adiponectin were used. Multivariable linear regression analyses with a subsequent backward procedure were carried out to demonstrate the independent relationship

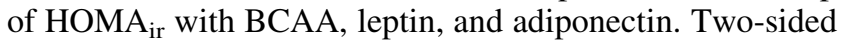
$P$-values $<0.05$ were considered significant.

\section{Results}

Seventy subjects (40 women and 30 men) participated, of whom 30 had normal fasting glucose, 25 had impaired fasting glucose, and 15 had T2DM (Table 1). BMI did not significantly vary across the three glucose tolerance groups $(P=0.56)$, but HOMAir was higher in T2DM subjects than in subjects with normal fasting glucose $(P<0.01)$. Total cholesterol $(P=0.05)$ was slightly lower in T2DM subjects than in subjects with normal fasting glucose. Plasma leptin and adiponectin levels were not significantly different between the three groups ( $P=0.86$ and $P=0.59$, respectively). BCAA did not significantly differ between the three glucose tolerance categories $(P=0.27)$, but tended to be higher in T2DM subjects versus the other subjects $(P=0.10)$. BCAA were higher in men $(452 \pm 82 \mu \mathrm{M})$ than in women $(372 \pm 74 \mu \mathrm{M}, P<0.001)$. Neither BCAA $(P=0.32)$ nor leptin $(P=0.80)$ and adiponectin $(P=0.22)$ levels were significantly different between the groups after adjustment for age and sex.

In univariate analysis carried out in all participants together, BCAA were associated with HOMAir $(r=0.46, P<0.001)$ and BMI $(r=0.40, P<0.001)$, and inversely with adiponectin $(r=-0.53, P<0.001)$ but not with leptin $(r=-0.08, P>0.05)$. In men, BCAA were correlated positively with HOMAir $(r=0.45$, $P=0.012)$ and BMI $(r=0.427, P=0.019)$, and inversely with adiponectin $(r=-0.554, P=0.001)$; the relationship with leptin was not significant $(r=0.32, P=0.084)$. In women, BCAA were similarly correlated positively with HOMAir $(r=0.457$, $P=0.003)$, BMI $(r=0.399, P=0.011)$, and leptin $(r=-0.33$, $P=0.037)$, and there was also a positive correlation with leptin ( $r=0.33, P=0.037$ ). Furthermore, in the whole group, HOMAir was positively correlated with BMI $(r=0.54, P<0.001)$ as well as with leptin $(r=0.35, P<0.001)$ and inversely with adiponectin $(r=-0.41, P<0.001)$. No correlation between leptin and adiponectin was observed $(r=0.06, P>0.05)$.

Multivariable linear regression analysis, adjusting for age, sex, diabetes status, and BMI, demonstrated that HOMAir was positively associated with BCAA (Table 2, model A). There was also an independent relationship of HOMAir with leptin taking account of BMI and adiponectin (Table 2, model B). Notably, when BCAA, leptin, and adiponectin were included together in the analyses, the positive relationships of HOMAir with BCAA and leptin remained present even independent of BMI (Table 2, model C). A similar age- and sex-adjusted relationship of HOMAir with BCAA, taking account of leptin and adiponectin, was found in multivariable analysis that did not include diabetes status 
Table 1. Clinical Characteristics, Insulin Resistance (Homeostasis Model Assessment), Plasma Leptin, Adiponectin, and Branched Chain Amino Acids in 70 Subjects with Varying Degrees of Glucose Tolerance (Normal Fasting Glucose, $N=30$; Impaired Fasting Glucose, $N=25$; Type 2 Diabetes Mellitus, $N=15$ )

\begin{tabular}{lcccc}
\hline & $N F G(\mathrm{n}=30)$ & $I F G(\mathrm{n}=25)$ & $T 2 D M(\mathrm{n}=15)$ & ANOVA P value \\
\hline Age (years) & $52 \pm 9$ & $56 \pm 9$ & $61 \pm 9^{\mathrm{a}}$ & 0.008 \\
Sex (men/women) & $11 / 19$ & $11 / 14$ & $8 / 7$ & 0.56 \\
Systolic blood pressure (mmHg) & $129 \pm 19$ & $131 \pm 21$ & $139 \pm 20$ & 0.29 \\
Diastolic blood pressure (mmHg) & $84 \pm 14$ & $80 \pm 8$ & $81 \pm 6$ & 0.31 \\
Body mass index $\left(\mathrm{kg} / \mathrm{m}^{2}\right)$ & $25.9 \pm 4.6$ & $25.0 \pm 3.0$ & $26.4 \pm 5.2$ & 0.56 \\
Glucose $(\mathrm{mM})$ & $5.3 \pm 0.5$ & $6.0 \pm 0.3$ & $8.3 \pm 2.1^{\mathrm{a}, \mathrm{b}}$ & $<0.001$ \\
Insulin $(\mathrm{mU} / \mathrm{L})$ & $6.3(4.3-8.6)$ & $6.0(4.6-8.3)$ & $6.1(4.5-9.7)$ & 0.60 \\
HOMAir, $(\mathrm{mU} / \mathrm{L}) \times(\mathrm{mM}) / 22.5$ & $1.5(1.0-2.1)$ & $1.7(1.2-2.4)$ & $2.3(1.6-3.8)^{\mathrm{a}}$ & 0.007 \\
Total cholesterol $(\mathrm{mM})$ & $5.8 \pm 1.1$ & $5.8 \pm 0.8$ & $5.0 \pm 0.9^{\mathrm{c}}$ & 0.02 \\
HDL cholesterol $(\mathrm{mM})$ & $1.5 \pm 0.4$ & $1.6 \pm 0.4$ & $1.6 \pm 0.5$ & 0.54 \\
Triglycerides $(\mathrm{mM})$ & $1.3(0.8-2.0)$ & $1.3(0.9-1.9)$ & $1.6(0.8-2.3)$ & 0.97 \\
Leptin $(\mu \mathrm{g} / \mathrm{L})$ & $6.8(3.2-22.7)$ & $5.9(3.3-23.7)$ & $5.9(1.3-31.4)$ & 0.86 \\
Adiponectin $(\mathrm{mg} / \mathrm{L})$ & $22.7(15.0-48.1)$ & $21.3(16.2-40.7)$ & $17.0(12.4-33.0)$ & 0.59 \\
BCAA $(\mu \mathrm{M})$ & $397 \pm 92$ & $398 \pm 73$ & $439 \pm 95$ & 0.27 \\
\hline
\end{tabular}

Data in mean $\pm \mathrm{SD}$ or in median (interquartile range).

${ }^{a} P<0.01$ from subjects with NFG.

${ }^{\mathrm{b}} P<0.05$ from subjects with IFG.

${ }^{\mathrm{c}} P \leq 0.05$ from subjects with NFG.

BCAA, branched chain amino acids; HOMAir, homeostasis model assessment; IFG, impaired fasting glucose; NFG, normal fasting glucose; T2DM, type 2 diabetes mellitus.

( $\beta=0.370, P=0.001)$, BMI $(\beta=0.275, P=0.012)$, or both $(\beta=0.332, P=0.004)$ (data not shown).

\section{Discussion}

As far as we are aware, our study is the first to delineate the relationship between BCAA and insulin resistance in the context of adipokines, in particular, leptin and adiponectin. As

Table 2. Multivariable Linear Regression Analysis Demonstrating Relationships of INSUlin Resistance (Homeostasis Model Assessment ) WIth

Plasma Branched Chain Amino Acids, Leptin, AND Adiponectin In 70 SubJECTS WITH VARYING

Degrees of Glucose Tolerance (Normal

FAsting Glucose, $N=30$; ImPaIREd Fasting Glucose, $N=25$; Type 2 Diabetes Mellitus, $N=15$ )

\begin{tabular}{|c|c|c|c|c|c|c|}
\hline & \multicolumn{2}{|c|}{ Model A } & \multicolumn{2}{|c|}{ Model B } & \multicolumn{2}{|c|}{ Model C } \\
\hline & $\beta$ & $\mathrm{P}$ & $\beta$ & $\mathrm{P}$ & $\beta$ & $\mathrm{P}$ \\
\hline Age & 0.005 & 0.96 & 0.038 & 0.72 & .016 & 0.88 \\
\hline Sex (men/ & -0.058 & 0.60 & 0.268 & 0.13 & 0.223 & 0.20 \\
\hline $\begin{array}{l}\text { wome } \\
\text { T2DM }\end{array}$ & 0.2 & & $\begin{array}{l}\mathbf{0 . 5 7 4} \\
0.280\end{array}$ & $<0$. & $\begin{array}{l}34 \\
66\end{array}$ & $\begin{array}{l}07 \\
07\end{array}$ \\
\hline (yes/no) & 0.252 & 0.011 & 0.320 & 0.001 & 0.281 & 0.003 \\
\hline BMI & $\begin{array}{l}0.411 \\
\mathbf{0 . 4 1 7}\end{array}$ & $\begin{array}{l}<0.001 \\
<\mathbf{0 . 0 0 1}\end{array}$ & 0.213 & 0.089 & 0.158 & 0.22 \\
\hline BCAA & 0.271 & 0.026 & & & 0.196 & 0.099 \\
\hline & 0.242 & 0.023 & & & 0.275 & 0.012 \\
\hline Leptin & & & 0.463 & 0.007 & 0.466 & $\begin{array}{r}0.006 \\
\mathbf{0} 001\end{array}$ \\
\hline & & & 0.744 & $<0.001$ & 0.640 & $<0.001$ \\
\hline Adiponectin & & & -0.210 & 0.075 & -0.146 & 0.232 \\
\hline
\end{tabular}

Variables with which HOMAir is independently associated by multivariable linear regression analysis with a subsequent backward procedure are shown in bold. Model A includes age, sex, T2DM, BMI, and BCAA. Model B includes age, sex, T2DM, BMI, leptin, and adiponectin. Model $\mathrm{C}$ includes age, sex, T2DM, BMI, BCAA, leptin, and adiponectin.

$\beta$, standardized regression coefficient; BMI, body mass index. expected from previously published literature, our study revealed a fairly strong relationship between BCAA and insulin resistance among subjects with varying degrees of glucose tolerance. Notably, in all subjects combined, BCAA were univariately correlated with insulin resistance, BMI, and adiponectin but not with leptin, even though leptin and adiponectin were each associated with insulin resistance. Our finding that insulin resistance was still related to BCAA, independent of diabetes status, BMI, leptin, and adiponectin, raises the possibility that BCAA and these adipokines may act, in part, by different mechanisms on insulin-mediated glucose metabolism. Our findings also suggest that adipose tissue dysfunction, as reflected by plasma levels of leptin and adiponectin, is unlikely the main pathogenic mechanism explaining the relationship of insulin resistance with circulating BCAA.

Obesity-associated plasma BCAA elevation levels have been proposed to indicate BCAA dysmetabolism, resulting in abnormal mitochondrial function, which in turn contributes to insulin resistance. ${ }^{3,4}$ Yet, it is still possible that increased circulating BCAA levels represent a mere marker of impaired insulin action. ${ }^{3}$ Of further interest, it is well recognized that leptin- and adiponectin-mediated processes are involved in the pathogenesis of insulin resistance as well; although the contributing molecular mechanisms appear to be at least, in part, different from that exerted by BCAA.,12,14,17 Importantly, leptin and adiponectin modulate low-grade inflammation and hence are thought to reflect adipose tissue dysfunction. ${ }^{14,16-18}$

Together with a direct effect of leucine, which makes part of the BCAA measurement in this study, on leptin metabolism, ${ }^{3}$ the well-documented relationships of insulin resistance with circulating BCAA, leptin, and adiponectin levels ${ }^{1,10,12}$ led us to explore the extent to which the relationship of insulin resistance with BCAA was explained by plasma levels of these adipokines. Our study shows that the relationship of insulin resistance with leptin and adiponectin is independent of BMI, in agreement with the role of these adipokines in adipose tissue dysfunction. ${ }^{17,18} \mathrm{~A}$ novel finding of the current report is that 
the relationship of insulin resistance with BCAA was independent of leptin and adiponectin also when taking BMI into account. Assuming that leptin and adiponectin are valid biomarkers of altered adipose tissue functionality, this result agrees with the hypothesis that the insulin resistance-BCAA association is not to a considerable extent driven by adipose tissue dysfunction. In view of the emerging role of the hepatokine, fetuin-A, in the pathogenesis of insulin resistance, ${ }^{22}$ and the observation that fetuin-A is associated with incident diabetes, even independent of adiposity measures, ${ }^{23}$ it is relevant to put the role of BCAA in diabetes development in the context of hepatokine dysregulation in future studies.

Several methodological issues should be considered. The cross-sectional design does not allow us to address the nature of the observed relationships. We cannot exclude the possibility that elevated BCAA are contributing to or that they are a result of insulin resistance and altered adipose tissue function. In addition, a limited number of T2DM subjects were included whose metabolic control was generally sufficient. Moreover, BMI did not significantly vary across the glucose tolerance groups. Participants did not receive hypoglycemic drugs or antihypertensive medication, thus avoiding confounding due to drug effects on insulin resistance. This likely explains why BCAA only tended to be higher in subjects with T2DM compared to those who were normoglycemic or had impaired fasting glucose. Lipid-lowering drugs were not used, explaining why subjects with only mild lipid abnormalities preferentially participated.

In conclusion, BCAA are associated positively with insulin resistance even when taking account of leptin and adiponectin diabetes and adiposity. This suggests that the relationship of insulin resistance with BCAA is at least, in part, independent of adipose tissue dysfunction.

\section{Authors' Contributions}

R.P.F.D. designed the study and analyzed the data; J.W.D. supplied the BCAA data; R.P.F.D., M.A.C., and J.W.D. interpreted the data; R.P.F.D. and M.A.C. wrote the article.

\section{Author Disclosure Statement}

M.A.C. and J.W.D. are employees of LabCorp. R.P.F.D reports no conflicts of interest.

\section{References}

1. Newgard CB. Interplay between lipids and branched-chain amino acids in development of insulin resistance. Cell Metab 2012;15:606-614.

2. Wurtz P, Soininen P, Kangas AJ, et al. Branched-chain and aromatic amino acids are predictors of insulin resistance in young adults. Diabetes Care 2013;36:648-655.

3. Lynch CJ, Adams SH. Branched-chain amino acids in metabolic signalling and insulin resistance. Nat Rev Endocrinol 2014;10:723-736.

4. Boulet MM, Chevrier G, Grenier-Larouche T, et al. Alterations of plasma metabolite profiles related to adipose tissue distribution and cardiometabolic risk. Am J Physiol Endocrinol Metab 2015;309:E736-E746.

5. Pedersen HK, Gudmundsdottir V, Nielsen HB, et al. Human gut microbes impact host serum metabolome and insulin sensitivity. Nature 2016;535:376-381.

6. Wang TJ, Larson MG, Vasan RS, et al. Metabolite profiles and the risk of developing diabetes. Nat Med 2011;17:448-453.
7. Floegel A, Stefan N, Yu Z, et al. Identification of serum metabolites associated with risk of type 2 diabetes using a targeted metabolomic approach. Diabetes 2013;62:639-648.

8. Lotta LA, Scott RA, Sharp SJ, et al. Genetic predisposition to an impaired metabolism of the branched-chain amino acids and risk of type 2 diabetes: A mendelian randomisation analysis. PLoS Med 2016;13:e1002179.

9. Shah SH, Crosslin DR, Haynes CS, et al. Branched-chain amino acid levels are associated with improvement in insulin resistance with weight loss. Diabetologia 2012;55:321-330.

10. Polyzos SA, Aronis KN, Kountouras J, et al. Circulating leptin in non-alcoholic fatty liver disease: A systematic review and meta-analysis. Diabetologia 2016;59:30-43.

11. Mather KJ, Goldberg RB. Clinical use of adiponectin as a marker of metabolic dysregulation. Best Pract Res Clin Endocrinol Metab 2014;28:107-117.

12. Caselli C. Role of adiponectin system in insulin resistance. Mol Genet Metab 2014;113:155-160.

13. Andrade-Oliveira V, Camara NO, Moraes-Vieira PM. Adipokines as drug targets in diabetes and underlying disturbances. J Diabetes Res 2015;2015:681612.

14. Beltowski J. Leptin and atherosclerosis. Atherosclerosis 2006;189:47-60.

15. Dallinga-Thie GM, Dullaart RP. Do genome-wide association scans provide additional information on the variation of plasma adiponectin concentrations? Atherosclerosis 2010;208:328-329.

16. Bluher M, Mantzoros CS. From leptin to other adipokines in health and disease: Facts and expectations at the beginning of the 21st century. Metabolism 2015;64:131-145.

17. Finucane FM, Luan J, Wareham NJ, et al. Correlation of the leptin: Adiponectin ratio with measures of insulin resistance in non-diabetic individuals. Diabetologia 2009;52:2345-2349.

18. Dullaart RP, Gruppen EG, Connelly MA, et al. A biomarker of inflammatory glycoproteins, is more closely related to the leptin/adiponectin ratio than to glucose tolerance status. Clin Biochem 2015;48:811-814.

19. Dullaart RP, de Vries R, van Tol A, et al. Lower plasma adiponectin is a marker of increased intima-media thickness associated with type 2 diabetes mellitus and with male gender. Eur J Endocrinol 2007;156:387-394.

20. Matyus SP, Braun PJ, Wolak-Dinsmore J, et al. NMR measurement of LDL particle number using the Vantera Clinical Analyzer. Clin Biochem 2014;47:203-210.

21. Jeyarajah EJ, Cromwell WC, Otvos JD. Lipoprotein particle analysis by nuclear magnetic resonance spectroscopy. Clin Lab Med 2006;26:847-870.

22. Stefan N, Haring HU. The role of hepatokines in metabolism. Nat Rev Endocrinol 2013;9:144-152.

23. Stefan N, Sun Q, Fritsche A, et al. Impact of the adipokine adiponectin and the hepatokine fetuin-A on the development of type 2 diabetes: Prospective cohort- and crosssectional phenotyping studies. PLoS One 2014;9:e92238.

Address correspondence to: Robin P.F. Dullaart, MD, PhD

Department of Endocrinology University Medical Center Groningen University of Groningen PO Box 30.001

Groningen $9700 \mathrm{RB}$ The Netherlands

E-mail: r.p.f.dullaart@int.umcg.nl 